DOI: 10.20472/TEC.2018.005.003

MICHAEL ELSDON

Northumbria University, United Kingdom

KRISHNA BUSAWON

Northumbria University, United Kingdom

ANNE VIGOUROUX

University of Nice Sophia Antipolis, France

RICHARD BINNS

Northumbria University, United Kingdom

IAN FORBES

Northumbria University, United Kingdom

VANESSA RAILLAND

INSA of Bourges, United Kingdom

\title{
A COMPARATIVE ASSESSMENT OF DELIVERY METHODS OF MATHEMATICS AND TECHNOLOGY ENHANCED LEARNING
}

\begin{abstract}
:
This paper is concerned with the delivery methods of mathematics in engineering subjects and the use of technology enhanced learning in higher education systems. Anecdotal evidence suggests that there is a lack of interest in mathematical modules by engineering students in recent years, a trend that appears to be growing. At the same time, many universities have invested heavily in e-learning tools in an effort to engage students. This report aims to investigate whether these tools are a support or barrier when teaching mathematics to engineering students, and to examine if they have had any effect on student engagement. The report also investigates student opinions of technology enhanced learning versus traditional methods of teaching.
\end{abstract}

\section{Keywords:}

assessment of learning, technology enhanced learning, delivery methods

JEL Classification: $121,129,120$ 


\section{Introduction}

In recent years there has been a continued decline in interest among young students in mathematics, science subjects and engineering subjects across Europe. With respect to undergraduate engineering graduates, a key requirement is a core understanding of mathematical concepts. However, the growing lack of interest in such subjects is a real concern for the quality of the industrial workforce. One would generally expect the contrary in the modern world where there is an easy access to IT services such as the internet where massive amounts of information and free tutorials are readily available. Furthermore, most educational institutions have invested a great deal of money on refurbishing their teaching environment by equipping them with various e-learning tools and resources. Power point and overhead projectors are now a common equipment that are found in almost all universities. Additionally, many universities have invested in various means allowing them to implement distance learning facilities to cater for the increasing amount of part-time students. The eLearning portal or Blackboard are commonly found in most UK universities. This has allowed lecturers and module tutors to deposit their lecture notes and provide feedback on assignments given to the students. The terms like wikis, doodle are very common vocabulary among young graduates. Many universities have also a center for teaching and learning where best practice in teaching and learning support are provided as a means of student and staff development. Based on these it is quite surprising to witness a decline of interest in mathematics amongst engineering under-graduates. A key concern, therefore when teaching under-graduate students modules that involve such heavy math content is how to engage students interest in such an important topic. The focus of this paper, is therefore to examine whether the use of emerging technology is a support or barrier when teaching mathematics to engineering students.

In the last few years, many project works have been devoted to define good practice in the design of web-based course at National level [1-2] or in the framework of the Socrates Programme [3-4]. All the content of these reports were not discussing the added value of a web based course versus a classical one for fundamental and technical module in various field of Electrical and Information Engineering. Besides that, problems of measurement of learning effectiveness of technology-based and conventional delivery methods should be taken into consideration as well [5]. At this point, evaluation and assessment should be not confused as they have a different role for the e-learning course [6]. 


\section{PILOT EXPERIMENT}

The current research seeks to understand why there is such a decline in student engagement with mathematical modules in engineering and whether eLearning tools themselves may be a contributory factor. We must note the changing student population. In previous years, the traditional student attending university could be described as being academically committed, motivated and highly interested in their studies. It has been suggested that this type of student learns regardless of the teaching style, and the student effectively 'teaches' themselves [7]. It could be argued that the 'traditional' student has a deep approach to learning and can access the material in numerous ways, but crucially via the medium of the lecture. In recent times, the increased popularity of higher education has meant that the 'traditional' student is no longer the norm. Although this student type still exists, students now come from a wide range of backgrounds, not necessarily via A-level route. Students may come from a non-traditional vocational background. Another group, who may not be as committed or motivated by the subject and simply sees the course as a passport to a well-paid job. With this in mind, the challenge for the lecturer is to adapt their teaching styles to accommodate the changing face of the student population. To this end, a significant investment has been made in various teaching technologies with the aim of increasing student engagement.

The aim of this study is therefore to investigate whether the use of such e-learning tools are a support or barrier, or are indeed responsible for such a decline in student engagement. The study also aims to find student views on the use of technology enhanced learning versus traditional teaching methods. For this purpose, a survey was carried out among Bachelor Degree Engineering students in order to obtain their opinion on the subject matter. The survey looks at the issue on the way that mathematics is delivered either by traditional methods (chalk and whiteboard markers) or by the use of eLearning tools (power point and overhead projectors). 
The survey used for pilot experiment is given in table 1 .

\section{Student Questionnaire}

\section{Name (optional):}

Gender: i) $M \quad$ ii) $F$

Academic Year:

Programme: BEng Electrical Engineering

Course/Module:

Nationality:

Encircle the answers that are most related to you:

Q1) I prefer when the module tutor (lecturer/teacher):

1. hands out the lecture notes in advance before the lecture

2. hands out the lecture notes at the beginning the lecture

3. does not hand out his lecture notes and lets me take lecture notes

Q2) When teaching in general, I prefer that the module tutor uses

1. only e-learning tools (power point or overhead projectors)

2. only traditional methods (whiteboard)

3. a mixture of both methods

Q3) When teaching the mathematics involved in DSP, I prefer that the module tutor uses
1. only e-learning tools (power point or overhead projectors)
2. only traditional methods (whiteboard)
3. a mixture of both methods

Q4) I feel intimidated when there are a lot of mathematical formulae displayed at once on a power point slide:
1. strongly agree
2. agree
3. disagree
4. strongly disagree

Q5) I prefer when the module tutor

1. derives the mathematical concepts and formulae step by step using the whiteboard

2. derives the mathematical concepts and formulae step by step using power point

3. derives the mathematical concepts and formulae step by step using either power point or the whiteboard

4. does not derives the mathematical concepts and formulae step by step but briefly explains the essential mathematical concepts and formulae by whatever means he likes.

Q6) I believe that my level in mathematics is
1. very good
2. good
3. average
4. poor 


\section{PILOT ANALYSIS}

The experiment has been set with a classroom involved in a bachelor degree of Electronic Engineering with the following characteristic:

- Results on ELearning Survey 1 -Year 2018

- Number of students: 212

- Nationality: French $=167$, Chinese $=19$, Moroccan $=12$, Other $=10$

Male: 149, Female: 63

The results on the Student Survey are displayed in the figures 1 to 6 .

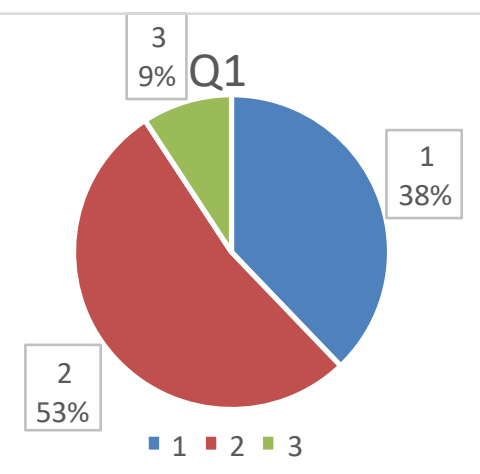

Figure 1: Student Survey: Question 1

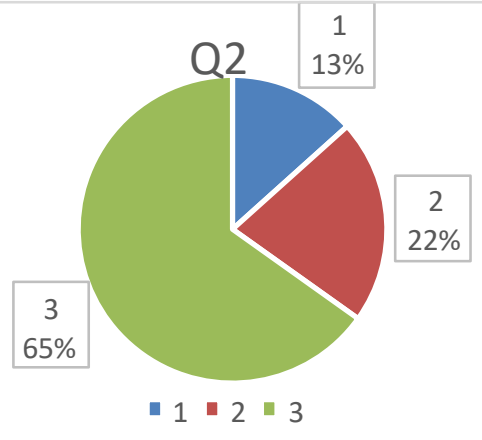

Figure 2: Student Survey: Question 2 


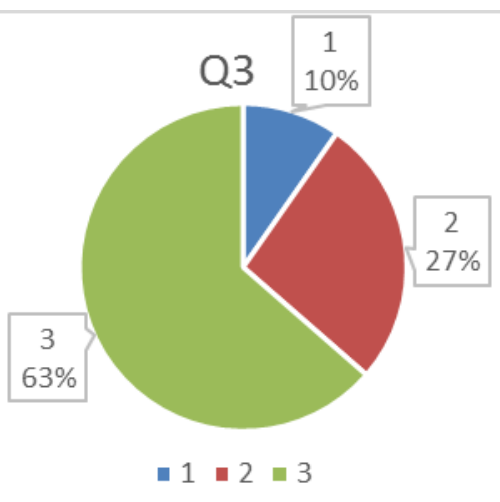

Figure 3: Student Survey: Question 3

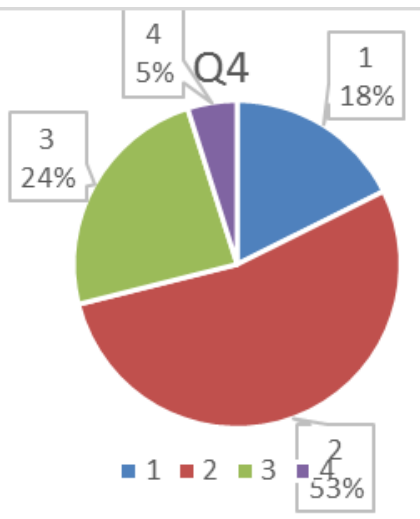

Figure 4: Student Survey: Question 4

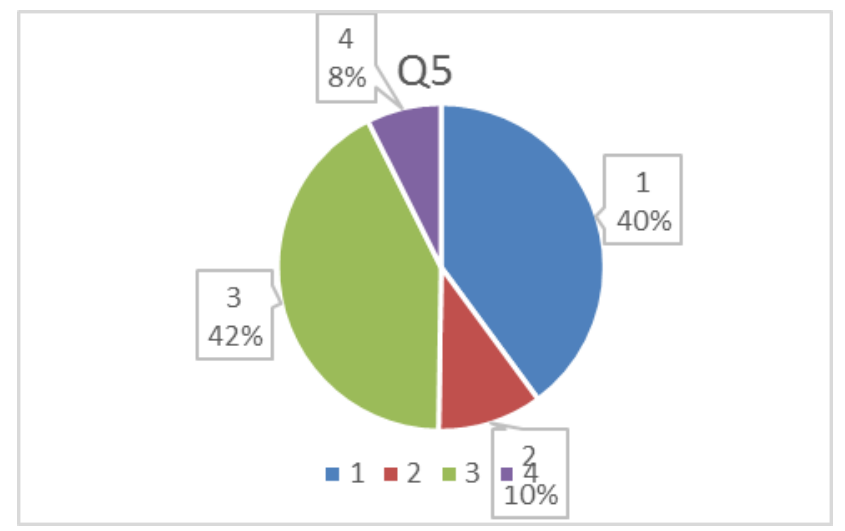

Figure 5: Student Survey: Question 5 


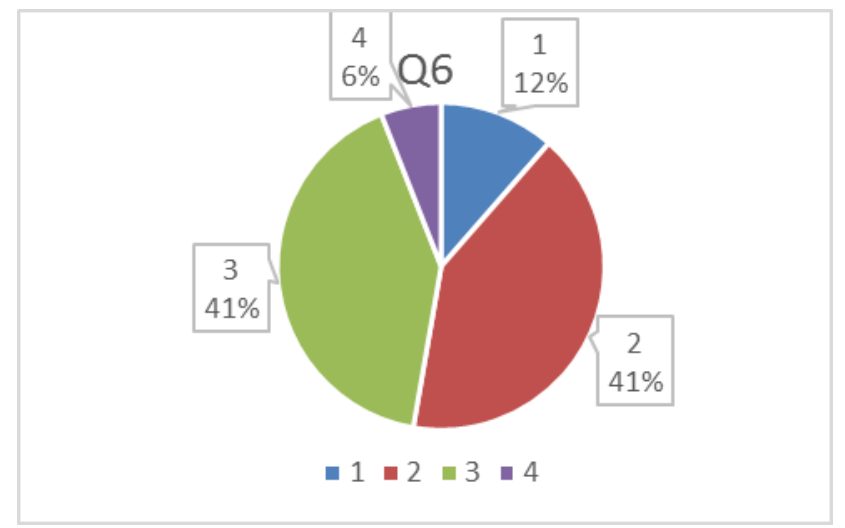

Figure 6: Student Survey: Question 6

Comments on ELearning survey:

Q1): The result suggests that the majority students are curious to know in advance about what they are going to be taught. They probably do not like the surprise element in teaching. For the module tutor or lecturer this might mean additional time should be given to prepare and print or copy their lectures by using any relevant tools. It is not surprising that students do not appreciate having to take notes for themselves. It would be an interesting study to find out educator's opinion on whether or not students should take notes. My feeling is that some educators would favor students taking notes themselves as they feel that this would help students learning. This would seem an outdated method of teaching, and something the author disagrees with. It would present more opportunities for learning if students were given material before class. This would allow for more deep discussion during teacher-student contact time.

Q2): Here the result clearly suggests that eLearning tools on their own are not an appropriate tool as far as teaching of any subject is concerned. However, their importance seems to acknowledge them as a useful teaching support medium. For the educational institutions, it means that a massive investment in eLearning media is not particularly necessary. It also suggests that the role of the module tutor as a professional and skillful pedagogue is required. It could be argued that a skilled teacher can achieve good performance regardless of the whether or not technology is used. The rationale for using technology should therefore be to only include it if it can be used effectively, something only a skilled teacher can do. From an educational viewpoint it would also be advantageous to utilize a wide range of e-learning and traditional teaching tools. This would allow us to cater for a number of different student learning styles. 
Q3): This result is also consistent with those obtained in Question 2. It does however suggest that Mathematics is really a distinct subject that needs to be treated separately and can't be taught by the use solely e-learning by itself. The majority of students state that only traditional methods (chalk, whiteboard) of teaching should be used when teaching mathematics. On first inspection, this would appear to be a key finding that suggests maths is a difficult subject to teach using purely electronic material. Conversely, it could also be argued it is not the technology, but the way in which the technology is used that is the key challenge.

Q4): This result is in accordance with the result given in Question 2. The display of numerous formulae by using eLearning tools is not a recommended idea while teaching mathematics. The results of this question could suggest that the majority of students see themselves as sequential who favor a step-by-step approach to learning, rather than global learners.

Q5): This result provides an answer as to what should be done when teaching mathematics. A step by step approach should be adopted when teaching mathematics. This would again suggest that engineering students see themselves as sequential learners. Not much importance seems to be attached as to whether traditional methods or eLearning tools are used to achieve this. However, the students still preferred the traditional method when it comes to teaching mathematics. A further avenue of study would be to see if there is any correlation between student's level of math and whether they see themselves as global or sequential learners.

Q6) Most students believed that they have an average or good level of mathematics.

\section{CONCLUSION}

It is hoped that through this survey, engineering educational institutions will rethink their policies when it comes to design effective teaching strategies and efficient allocation of budgets in relation to the delivery of engineering subjects. The greatest effect on students' education is the teaching method. If we have a skilled teacher who is able to explain key concepts - he/she can do this regardless of the technology involved. You could argue that e-learning tools do not imply an effective delivery. However, if used correctly they can enhance delivery. The key question that needs to be addressed when investing in technology is whether the teacher is able to use this effectively. This paper 
has highlighted initial results from a small pilot study. There are a number of avenues for further research to enhance this study further. These include investigating the teaching methods students from different nationalities, level of study and mathematical ability. It would also be interesting to compile a similar survey to find out the views of educators.

\section{REFERENCES}

Sarah Knight, Effective Practice with e-Learning, A good practice guide in designing for learning, JISC Development Group Bristol, UK.

John Milne and Emily Dimock, e-Learning Guidelines, Guidelines for the support of learning in New Zealand tertiary institutions, http://elg.massey.ac.nz, June 2006.

ESOFEL, Project Effective Strategies for Open, Flexible E-learning. A Framework for Effective Online Tutoring A European partnership project - West Lothian College, UK Greta du Velay, France, Environmental School Cesis, Latvia.

ICT Cluster and the Danish Technological Institute Using e-Learning in Teacher Training - Good Practice Cases, Compendium on Good Practice Cases on e-Learning' ICT Cluster edition.

Ernest H. Joy II, Federico E. Garcia, Measuring Learning Effectiveness: A New Look at No-SignificantDifference Findings. JALN Volume 4, Issue 1, pp. 33-39, June 2000,

Rob Phillips (editor), John Bain, Carmel McNaught, Mary Rice, David Tripp, Handbook for Learning-centred Evaluation of Computer-facilitated Learning Projects in Higher Education, 2000

Biggs and Tang, 'Teaching for Quality Learning at University', 2000. 